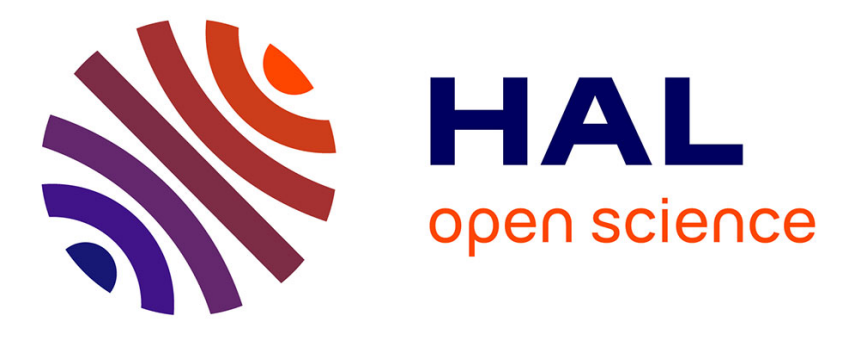

\title{
MAX: Development of a Laue diffraction lens for nuclear astrophysics
}

\author{
N. Barriere, P. von Ballmoos, G. Skinner, B. Smither, P. Bastie, E. Hinglais, \\ N. Abrosimov, J.M. Alvarez, K. Andersen, P. Courtois, et al.
}

\section{- To cite this version:}

N. Barriere, P. von Ballmoos, G. Skinner, B. Smither, P. Bastie, et al.. MAX: Development of a Laue diffraction lens for nuclear astrophysics. Fourth International Conference on New Developments in Photodetection, Jun 2005, Beaune, France. Elsevier, 567, pp.333-336, 2006, 10.1016/j.nima.2006.05.150 . in2p3-00112346

\section{HAL Id: in2p3-00112346 \\ https://hal.in2p3.fr/in2p3-00112346}

Submitted on 21 Nov 2006

HAL is a multi-disciplinary open access archive for the deposit and dissemination of scientific research documents, whether they are published or not. The documents may come from teaching and research institutions in France or abroad, or from public or private research centers.
L'archive ouverte pluridisciplinaire HAL, est destinée au dépôt et à la diffusion de documents scientifiques de niveau recherche, publiés ou non, émanant des établissements d'enseignement et de recherche français ou étrangers, des laboratoires publics ou privés. 


\title{
MAX: Development of a Laue diffraction Lens for nuclear astrophysics
}

N. Barriere ${ }^{(1)}$, P. von Ballmoos ${ }^{(1)}$, G. Skinner ${ }^{(1)}$, B. Smither ${ }^{(2)}$, P. Bastie $^{(3)}$, E. Hinglais ${ }^{(4)}, N$. Abrosimov $^{(5)}$, J. M. Alvarez ${ }^{(6)}$, K. Andersen ${ }^{(7)}$, P. Courtois ${ }^{(7)}$, H. Halloin ${ }^{(8)}$, M.Harris ${ }^{(1)}$, M. Hernanz ${ }^{(6)}$, J. Isern ${ }^{(6)}$, P. Jean ${ }^{(1)}$, J. Knödlseder ${ }^{(1)}$, P. Ubertini ${ }^{(9)}$, G. Vedrenne ${ }^{(1)}$, G. Weidenspointner ${ }^{(1)}$, C. Wunderer ${ }^{(10)}$

(1) Centre d'Etude Spatiale des Rayonnements, Université Paul Sabatier - Toulouse III, 9, avenue du Colonel Roche, BP 4143 - 31028 Toulouse Cedex 4 -France

(2) Argonne National Laboratory, 9700 S. Cass Avenue, Argonne, IL 60439. - USA

(3) Laboratoire de Spectrométrie Physique, 38402 Saint Martin d'Hères - France

(4) Centre National d'Etudes Spatiales, 18 avenue Edouard Belin, 31401 Toulouse - France

(5) Institut für Kristallzüchtung, Max-Born-Strasse 2, D-12489 Berlin - Germany

(6) Institut d'Estudis Espacials de Catalunya, Despatx 201 Edifici Nexus C/ Gran Capità, 2-4, 8034 Barcelona - Spain

(7) Institut Laue Langevin, 6, rue Jules Horowitz BP 156 - 38042 Grenoble Cedex 9 - France

(8) Fédération de Recherche APC, Collège de France, 11 place Marcelin Berthelot, 75231 Paris - France

(9) Istituto di Astrofisica Spaziale e Fisica Cosmica, Via del Fosso del Cavaliere 100, 00133 Roma - Italy

(10) Space Sciences Laboratory, University of California, Berkeley, CA 94720-7450 - USA

* Corresponding author: Phone:+33561557569; Fax:+33561556651; barriere@cesr.fr

\begin{abstract}
The next generation of instrumentation for nuclear astrophysics will have to achieve an improvement in sensitivity by a factor of 10-100 over present technologies. With the focusing gammaray telescope MAX we take up this challenge and propose to combine the required sensitivity with high spectral and angular resolution, and the capability to measure the polarization of the photons. MAX is a space-borne crystal diffraction telescope, featuring a broad-band Laue lens optimized for the observation of compact sources in two wide energy bands of high astrophysical relevance. Gamma rays will be focused from the large collecting area of a crystal diffraction lens onto a very small detector volume. As a consequence, the signal to background ratio is greatly enhanced, leading to unprecedented sensitivities.
\end{abstract}

PACS: 95.55.Ka; 29.30.Kv; 61.10.-i;

Key words: Instrumentation: gamma-ray Laue lens; Gamma-ray astrophysics; mosaic crystals;

\section{Introduction}

Since its launch in 2002, the INTEGRAL (INTErnational Gamma RAy Laboratory) observatory is providing a global overview of the soft gamma ray. New and exciting gamma-ray sources are being detected, and now there is a growing interest in the capability of performing deeper, more focused investigations. To improve our und erstanding of the most violent phenomena of the Universe, the next generation of instruments will have to achieve a gain in sensitivity of $10-100$ over present technologies.

To reach this goal, the concept of a gamma-ray telescope featuring a crystal diffraction lens is proposed. Compared to ex isting instruments relying on inelastic interaction processes - geometric optics (shadowcasting in modulating aperture systems) or quantum optics (kinetics of Compton scattering) - focusing allows to decouple the collecting area from the sensitive area. Since in space the background count rate in a gamma-ray detector is roughly proportional to its volume, focusing from a large collecting area onto a small detector leads to high signal to noise ratios, and so to high sensitivities.

MAX lens is based on Bragg diffraction in the volume of crystalline materials. A prototype of such a Laue lens, called CLAIRE, has already been realized in a collaboration CESR - CNES (the 
Franch space Agency), it has demonstrated the feasibility of this concept [1].

In this paper, a brief review of the principle of a Laue lens is given, followed by a description of the MAX mission. We also present a brief history of the R\&T program CLAIRE, and describe ongoing MAX R\&D for the development of a space borne Laue lens.

\section{The principle of Laue lenses}

In a crystal diffraction lens, crystal tiles are positioned such that they diffract the incident radiation of a certain energy onto a common focal spot according to the Bragg relation $2 d_{\mathrm{hkl}} \sin \left(\theta_{\mathrm{B}}\right)=$ $n \lambda$ as illustrated in Figure 1. This formula links a wavelength $\lambda$ to the Bragg angle $\theta_{\mathrm{B}}$ (diffraction angle) for a particular set of crystalline planes $[h k l]$ in the crystal and for a given diffraction order $n$.

Crystals suitable for a Laue lens must have a non-zero angular acceptance, i.e. a given wavelength must be diffracted for a non-zero range of incident angles. Such crystals are called mosaic crystals because they are well described by a juxtaposition of independent, and slightly differently, oriented small single crystals - the crystallites - as in a mosaic. The mosaic spread of a crystal is described by the mosaicity, which is given by the FWHM of the distribution function of the crystallite orientations.

A crystal angular acceptance $\Delta \theta$ is related to its energy bandpass $\Delta \lambda$ through the relation $\Delta \lambda / \lambda \approx$ $\Delta \theta / \theta$ derived from the Bragg relation. A mosaic crystal thus diffracts an energy bandpass $\Delta \mathrm{E}$ centred on a value which depends to the crystal's radial position on the lens (since the focal length $F_{\infty}$ is fixed, the radius $r$ imposes the diffraction angle): $\Delta \mathrm{E} \approx\left(2 F_{\infty} / r\right) \mathrm{E} \Delta \theta$.

Two subclasses of crystal diffraction lenses can be identified - narrow bandpass Laue lenses and broad bandpass Laue lenses. Narrow bandpass Laue lenses use a different set of crystalline planes $[h k l]$ for every ring in order to diffract photons in only one energy band centred on an energy E1 = E2 (Figure 1). Broad bandpass Laue lenses use one or very few sets of crystalline planes - typically the lowest order planes [111] or [200] which diffraction efficiency is optimum. Since several adjacent rings use the same set of planes, each focuses a slightly different energy because of the varying radii. This type of lens can cover a broad en ergy band. However, if a large geometric area is required, Laue lenses involve very long focal lengths because of the small Bragg angles values.

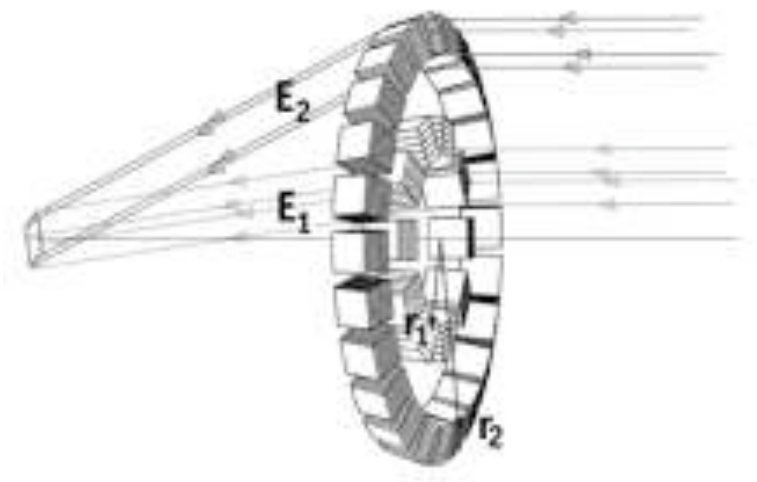

Figure 1. The basic design of a crystal diffraction lens.

\subsection{Lens features and mission objectives}

\section{The MAX mission}

The MAX mission is a space borne gamma-ray telescope consisting of two satellites flying in formation in order to achieve $86 \mathrm{~m}$ of focal length between the Laue lens and the detection plane. In the current design, the lens is made of 7870 copper and germanium crystal tiles of $1.5 \mathrm{~cm} \times 1.5 \mathrm{~cm}$, distributed on 24 concentric rings with radii ranging from 56 to $80 \mathrm{~cm}$ and from 96 to $112 \mathrm{~cm}$ (Figure 3 ). The mosaicity of the crystals is 30 arcsec, their total weight is about $120 \mathrm{~kg}$. 
The resulting lens focuses simultaneously in two broad energy bands corresponding to the scientific objectives of the mission, i.e. the study of Type Ia supernovae (SN) and compact objects. The bandpasses of $10 \mathrm{Cu}$ rings and $3 \mathrm{Ge}$ rings superimpose to cover an energy band from 800 to 900 $\mathrm{keV}$, with the aim to observe the $847 \mathrm{keV}$ and $812 \mathrm{keV}$ nuclear lines emitted by SN Ia. The effective area is $500 \mathrm{~cm}^{2}$ at $847 \mathrm{keV}$. A sensitivity of $\sim 10^{-6} \mathrm{~cm}^{-2} \mathrm{~s}^{-1}$ to broadened gamma-ray lines allows to expect $\sim 5$ observations of SN Ia per year, with in distances up to $50 \mathrm{Mpc}$ [2].

The second energy band is centred on $500 \mathrm{keV}$ in order to observe the electron - positron annihilation line at $511 \mathrm{keV}$. A significant numbers of positrons is expected to be released from compact objects, both galactic and extragalactic, such as microquasars, X-ray binaries, active galactic nuclei, solar flares and the high en ergy afterglow from gamma-ray bursts. Here the bandpasses of 9 $\mathrm{Cu}$ rings and $2 \mathrm{Ge}$ rings combine to cover an energy band from 450 to $540 \mathrm{keV}$, with a cumulated effective area of $725 \mathrm{~cm}^{2}$ at $511 \mathrm{keV}$. In this bandpass, the $478 \mathrm{keV}$ decay line from ${ }^{7} \mathrm{Li}$ in classical novae constitutes another important objective.

\subsection{Detector features}

The baseline detector for MAX is a stack of planar Ge detectors using orthogonal strips, cooled by a passive radiator and actively shielded by BGO scintillators. Alternative solutions include i) a single high purity germanium detector, ii) an efficiency optimized narrow field of view Compton camera featuring Si strips and CdTe pixels [3] [4], and iii) an array of low temperature calorimeters (detection of phonons produced by the impinging photons [5]).

The advantages of using a detector providing localization of the interactions are multiple: besides following the excursions of the focal spot across the detector plane, such a system allows the simultaneous measurement of signal and background. Most importantly, in a system with 3dimensional event localization, a significant further background reduction can be achieved by reconstructing the arrival direction of the photons using Compton kinematics, which allows rejecting photons not coming from the lens direction.

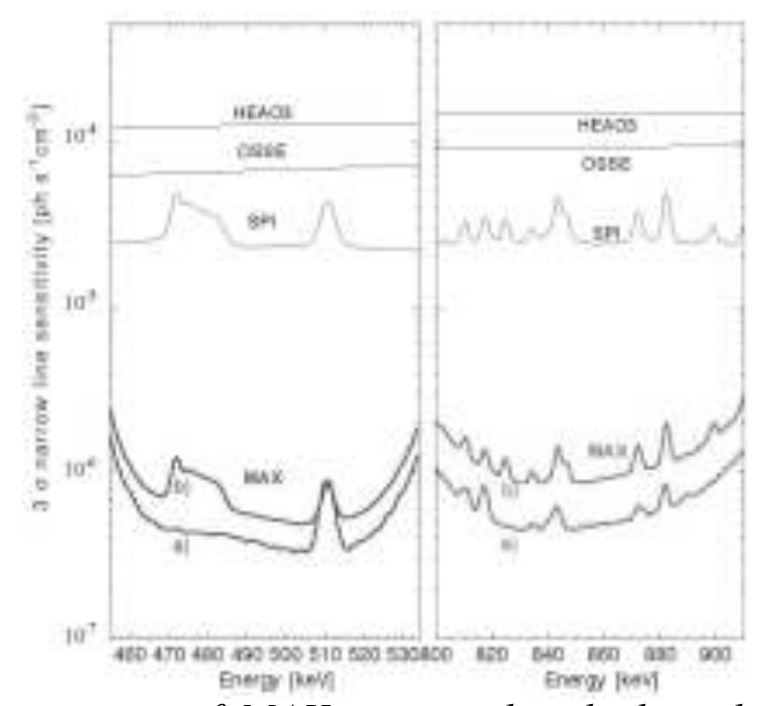

Figure 2. $3 \sigma$ narrow line sensitivity of MAX compared with the achieved sensitivities of earlier missions. Observation time is $10^{6}$ seconds. The MAX performance is based a) on the background of the TGRS Ge detector, scaled for background reduction using the Compton kinematics, and $b$ ) on the measured background of the INTEGRAL/SPI Ge detectors scaled to match the area of the lens focal spot.

\subsection{Sensitivity estimates}

The modelled narrow line sensitivity of MAX in each energy band is shown in Figure 2. Estimates are based on the diffraction efficiency given by the Darwin model, in agreement with the 
diffraction efficiencies measured for the lens prototype CLAIRE (see below). The background of the baseline detector (a) is a semi-empirical model based on the measured background from the unshielded TGRS ${ }^{1}$ [6] Ge detector and scaled for background reduction using Compton kinematics. Option (b) uses the specific background rate of an INTEGRAL/SPI Ge detector [7] scaled to match the area of the lens focal spot.

\subsection{Imaging Capabilities}

Although a crystal lens telescope is not a direct imaging system, the spatial response does depend on the source position in the field of view. For an on-ax is point source, the response is a 'focal spot' centred on the optical axis of the lens. But for an off-axis source, this peak becomes a circular ridge with a radius that depends on the off-ax is angle and with the position of the most intense point being given by a straight line from the source through the centre of the lens (as an optical lens).

The capability of Laue lenses to resolve possible $\mathrm{e}^{-}-\mathrm{e}^{+}$annihilation sources associated with the radiojets of the microquasar $1 \mathrm{E} 1740-29$ at $511 \mathrm{keV}$ has been demonstrated by extensive simulations $[8]$.

\section{The R\&D towards a spaceborne Laue Lens}

\subsection{CLAIRE}

As a first step, a ground-based prototype of a narrow band Laue lens system was built and successfully tested. Diffraction efficiencies of individual Ge crystals, measured at the Advanced Photon Source synchrotron at Argonne National Laboratories agree with what is expected from the Darwin model (efficiencies of $20 \%$ to $31 \%$ according to energy and crystal planes) [8]. The next step towards a space borne crystal lens telescope was to demonstrate a diffraction lens under space conditions and with an astrophysical target. This objective has been realized between 1999 and 2003 with the R\&D project CLAIRE. The CLAIRE lens consists of $556 \mathrm{Ge}_{\mathrm{x}}-\mathrm{Si}_{1-\mathrm{x}}$ mosaic crystals mounted on the eight rings of a $45 \mathrm{~cm}$ diameter titanium frame, and focusing $170 \mathrm{keV}$ photons onto a spot of $1.5 \mathrm{~cm}$ diameter at a distance of $279 \mathrm{~cm}$ from the lens.

Results of the CLAIRE project validate the theoretical models and demonstrate the potential of a gamma-ray lens for nuclear astrophysics (see [1] for a thorough discussion of the CLAIRE project).

\subsection{Lens design}

A first design of the MAX lens (Figure 3) has been performed in order to study thermo-elastic deformations. The design is based on the ALCATEL structural concept: A main cen tral beam supports 32 independent modules on which the individual crystals are attached. The central beam homogenizes the temperature and transmits mechanical efforts to the satellite structure through four titanium links. Due to the penetrating character of gamma rays it is possible to put the lens in a multilayer insulation (MLI) cocoon to passively insure sufficient thermal stability. Whatever the orientation of the sun, temperature excursions will stay within a range of $\sim 3 \mathrm{~K}$ around the nominal temperature.

A more constraining specification concerns out of plane thermo-elastic deformations of the lens. To

satisfy the Bragg condition for incident photons on its 30 arcsec mosaicity crystals, the crystal orientation will therefore have to be precise to within $\sim 10$ arcsec with respect to the lens optical ax is. This angular constraint translates in a warping of $100 \mu \mathrm{m}$ over the $2.24 \mathrm{~m}$ lens frame. Preliminary studies led by the CNES/PASO study group have shown that $\mathrm{a} \pm 2 \mathrm{~K}$ thermal control is essential to

\footnotetext{
${ }^{1}$ TGRS: Transient Gamma-Ray Spectrometer onboard the spacecraft WIND.
} 
satisfy the above deformation specifications - a requirement that is easily attainable [9].

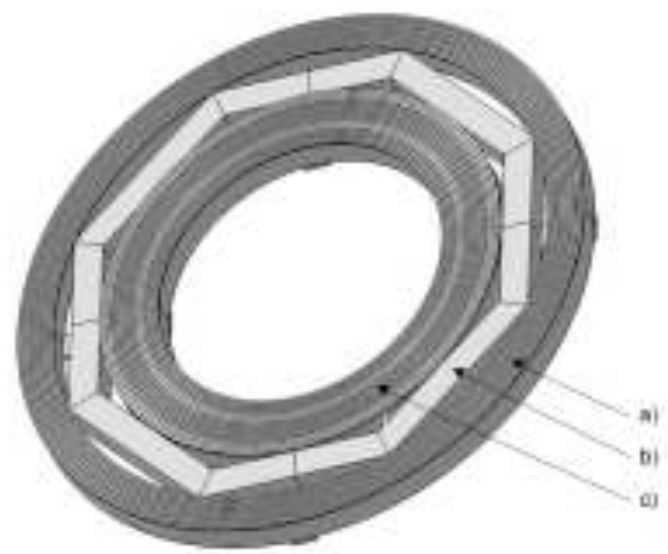

Figure 3: First design of MAX lens; a) the 9 outer $C u$ rings divided in 16 independent sections; b) the $\mathrm{SiC}$ or CeSiC main beam which support the 32 modules and c) inner $13 \mathrm{Cu}$ and Ge rings also divided in 16 sections.

\subsection{Second generation crystal}

Another crucial issue for MAX development is the lens diffraction efficiency. While CLAIRE's overall peak efficiency was $\sim 10 \%$, some of the 556 crystals have been measured with a peak efficiency of $\sim 20 \%$, i.e. close to the theoretical maximum of $\sim 25 \%$ for our mosaic crystals (taking into account absorption).

There are two reasons for CLAIRE's relatively low average efficiency. Firstly, each of the eight rings uses a different set of crystalline planes - the theoretical peak efficiencies range from $25 \%$ at best ([111] crystalline planes) down to less than 10\% ([333] crystalline planes). Secondly, the performance of individual crystals varies strongly from crystal to crystal, even in the same ring.

Since MAX is a broad-band lens, it is using only the lowest diffraction orders to achieve the highest theoretical efficiency. On other hand, since the time when CLAIRE's crystals have been grown, the reproducibility of high quality $\mathrm{Ge}_{\mathrm{x}} \mathrm{Si}_{1-\mathrm{x}}$ mosaic crystal has greatly improved. As an alternative, germanium composite crystals are currently being investigated: These are stacks of singlecrystal germanium wafers, that are slightly misoriented as are crystallites in a mosaic crystal. This technique presents two advantages with respect to mosaic crystals: single crystal wafers are easily reproducible and it's possible to optimize the thickness of each wafer. Preliminary experiments at the diffractometer of ILL (Grenoble, France) have shown a reflecting power comparable to that obtained with the best of CLAIRE's mosaic crystals [10]. A run at the European Synchrotron Radiation Facility (ESRF) scheduled for November 2005 will bring new clues about the quality of this emerging technique.

\section{Summary}

The R\&D program led by our collaboration - particularly the CLAIRE mission - has demonstrated the feasibility of a space borne Laue lens for nuclear astrophysics. The concept of a gamma-ray lens, which seemed unrealizable a few years ago, is now studied thoroughly in various laboratories.

Despite the fact that MAX needs two spacecraft flying in formation, and the rigorous requirements on thermo-elastic stability of the lens, the recent pre-phase A study of the French Space Agency CNES [9] has established the feasibility of MAX with present technologies. 
With its Laue lens consisting of $\mathrm{Cu}$ and Ge crystals rings MAX promises a breakthrough for the study of point sources at gamma-rays energies by combining narrow line sensitivities better than $10^{-6}$ $\mathrm{cm}^{-2} \mathrm{~s}^{-1}$ and high energy resolution $(\mathrm{E} / \mathrm{dE} \sim 500)$.

\section{References}

[1] Halloin H. 2003, Phd Thesis, University Toulouse III, www.cesr.fr $\sim$ pvb/MAX/publis/Diss_Halloin_03.pdf

[2] Leising M, Exp. Astrophys. 2006 (to be published)

[3] Takahashi T., 2004, New Astronomy Reviews, 48, 269-273

[4] Limousin O. et al. 2003, NIM A, 504, 24-37

[5] Giuliani A. 2001, Proc. SPIE Vol. 4507, 203

[6] Weidenspoin tner et al., 2005, ApJS 156, 69 - 91

[7] Jean P. et al., 2003, Astron.Astrophys., 411, L107

[8] Kohnle, A., 1998, Phd Thesis, University Toulouse III, www.cesr.fr pvb/MAX/publis/Diss Kohnle 98.pdf

[9] Hinglais E. et al., Exp. Astrophys 2006, (to be published)

[10] Barriere N. et al., Exp Astrophys 2006, (to be published) 